\title{
BESIII future prospects in exotic hadron spectroscopy
}

\author{
Kai ZHU ${ }^{1, a}$ \\ On behalf of the BESIII collaboration \\ ${ }^{1}$ Institute of High Energy Physics, CAS, Beijing, China
}

\begin{abstract}
Recent BESIII results on exotic hadron candidates, the heavy charmonium-like states, will be reviewed, as well as a short introductions to the BEPCII collider and the BEESIII detector. Future BESIII running plans, specially the physics program for the exotic hadron spectroscopy, will be discussed.
\end{abstract}

\section{Introduction of BESIII and BEPCII}

The Beijing Electron Positron Collider II(BEPCII) [1] is a double-ring $e^{+} e^{-}$collider with reached peak luminosity of $10^{33} \mathrm{~cm}^{-2} \mathrm{~s}^{-1}$ and beam current of $0.93 \mathrm{~A}$ in the $\tau$-charm energy region. The BESIII detector [2], operating at the BEPCII collider, is a general purpose spectrometer with a geometrical acceptance of $93 \%$ of $4 \pi$ stereo angle. It is composed by four main components: a multi-layer drift chamber (MDC) with a momentum resolution of $0.5 \%$ at $1.0 \mathrm{GeV} / \mathrm{c}$ in a $1.0 \mathrm{~T}$ magnetic field and a $d E / d x$ resolution better than $6 \%$ at the minimum ionization energy, a time-of-flight (TOF) detector constructed of $5 \mathrm{~cm}$ thick plastic scintillators providing a $2 \sigma K / \pi$ separation for momentum up to $1.0 \mathrm{GeV} / \mathrm{c}$, an electromagnetic calorimeter (EMC) in a cylindrical barrel structure and two end-caps providing deposited energy measurement of photon with resolution $2.5 \%(5 \%)$ at $1.0 \mathrm{GeV}$ in the barrel (end-caps), and a muon counter (MUC) consisting of $1000 \mathrm{~m}^{2}$ resistive plate chambers (RPCs) in nine barrel and eight end-cap layers. More details of the BESIII detector can be found in Ref. [2].

BESIII works in the $\tau$-charm energy region with abundant potential physics such light hadron, charmonium, charm, R-value and QCD, and new physics. Among them, the exotic hadrons may be the most exciting discoveries. Usually meson and baryon, the so called normal hadrons, take quark components that are quark anti-quark pair and three quarks, respectively. However, QCD allows states with more complex compositions such as penta-quark (five quarks), H-dibaryon (six quarks), tetraquark (four quarks), glueball, hybrid, molecule, etc., even not explicitly and definitely confirmed by experiments yet. These new hadron forms are exotic hadrons, or called as XYZ particles. Since 2012, the BESIII collaboration has systematically investigated the charmonium-like XYZ particles and made many important measurements. And it will go on this exploration with more data accumulated in this energy region. In this talk, I shall fast review the obtained exotic hadron results at BESIII so far, discuss what we know/what we don't know but want to know, data taking plan for XYZ particle study at BESIII, and what can be expected in the near future.

ae-mail: zhuk@ihep.ac.cn 


\section{Recent exotic hadron results from BESIII}
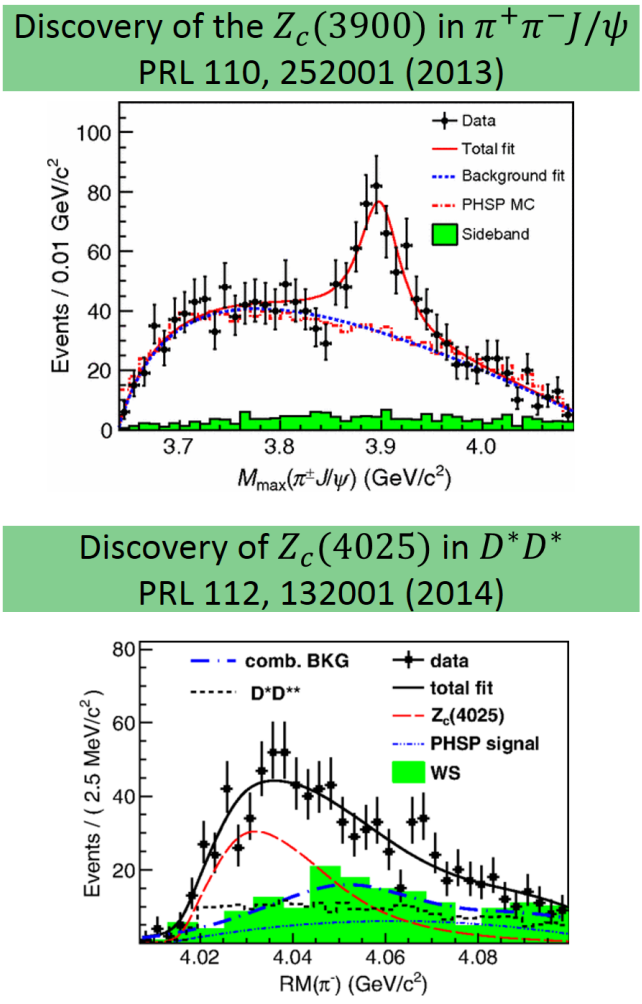
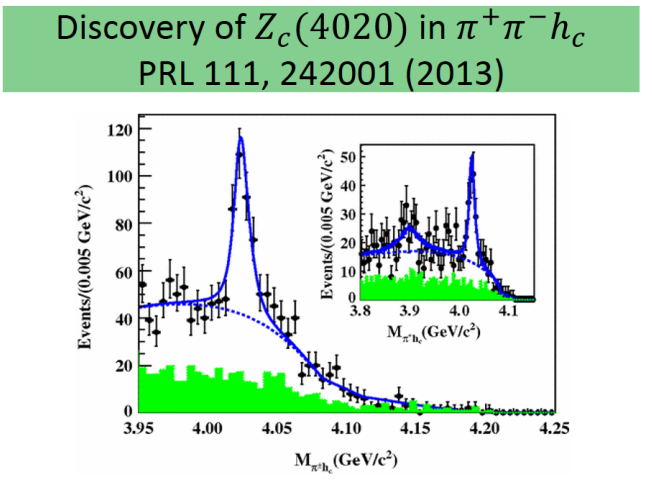

\section{Observation of $e^{+} e^{-} \rightarrow \gamma X(3872)$ PRL 112, 092001 (2014)}

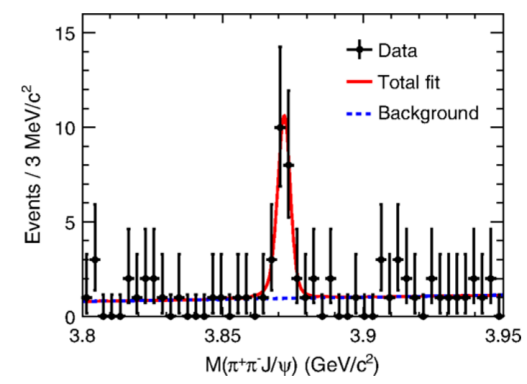

\section{Discovery $Z_{c}(3885)$ in $D D^{*}$ model \\ PRL 112, 022001 (2014)}
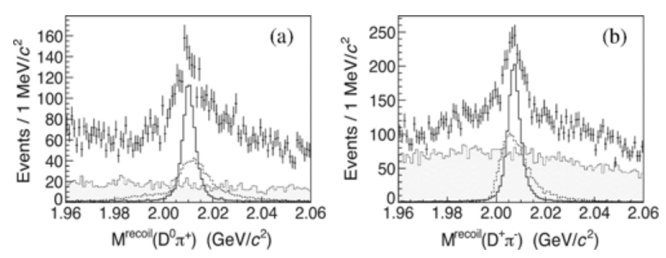

Figure 1. BESIII exotic hadron paper released in 2013.

Before talk about the future, let's take a look at the history of BESIII. The building of BEPCII and BESIII has been completed in 2007, and after commissioning the first $\psi(3686)$ event is observed at BESIII in 2008. In 2009 and 2010, large luminosity of data samples at the $\psi(3686), J / \psi$ and $\psi(3770)$ peaks have been collected. In 2011, 481 $\mathrm{pb}^{-1}$ data has been collected at the center mass energy (CME) 
$4.009 \mathrm{GeV}$, and this is the first bunch of data sample for exotic hadron study. In 2012, BESIII has accumulated more data at $\psi(3686)$ and $J / \psi$ peaks. Also in the same year, the first paper at BESIII for the exotic hadron is released, that is the measurement of the cross section of $\eta J / \psi$ at $\sqrt{s}=4.009 \mathrm{GeV}$. In this talk, the time stamp is according to the date when the paper is submitted to the arXiv e-print service.
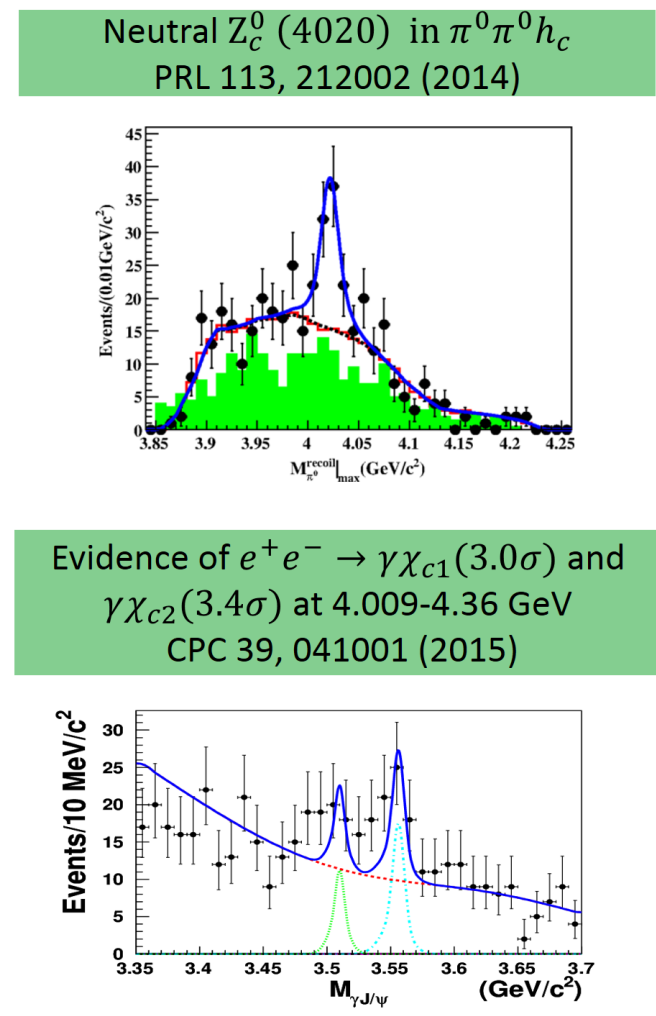

Figure 2. BESIII exotic hadron paper released in 2014.

\section{Discovery of a peak in $e^{+} e^{-} \rightarrow \omega \chi_{c 0}$ PRL 114, 092003 (2015)}
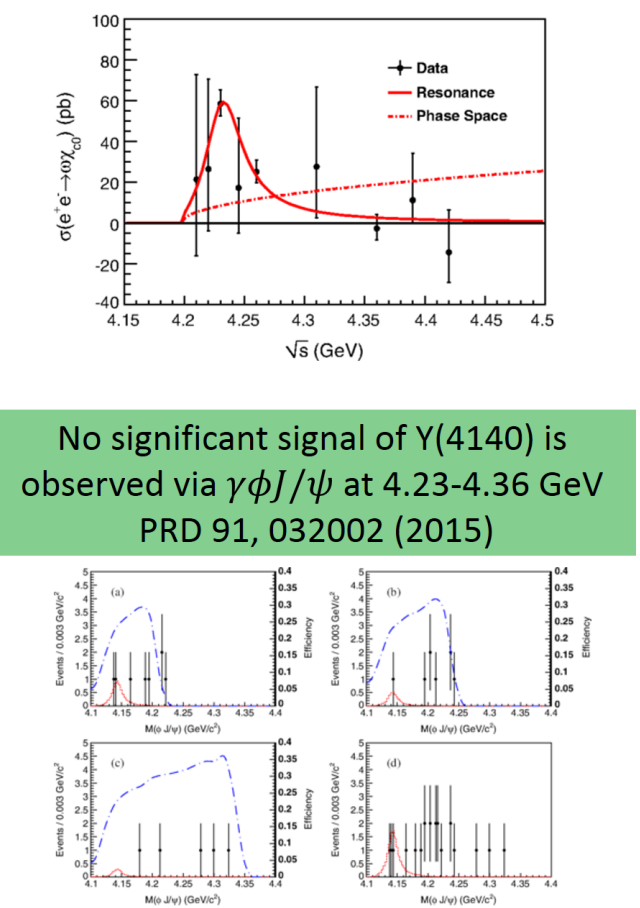

In 2013, more scan data for exotic hadron study, $2.9 \mathrm{fb}^{-1}$ from 4.23 to $4.6 \mathrm{GeV}$, has been collected at BESIII. And in this year, a paper based on analysis of $\pi^{+} \pi^{-} J / \psi$ final states has been released, that reported first time of the observation of $Z_{c}(3900)$ [3]. The nature of this newly found resonance is a myth, since it couples strongly to $c \bar{c}$ and has charge, so it must take at least four quarks. Many suggestions and models have been proposed to explain this state, such as $D D^{*}$ molecule, tetra-quark, cusp/threshold effect, or mixing, etc. But none of them is accepted throughout the community yet. Soon after that, BESIII published the discovery of another $Z$ resonance, $Z_{c}(4020)$, found in the $\pi^{+} \pi^{-} h_{c}$ final states with the data taken at $4.26 \mathrm{GeV}$ [4], as well as two $Z_{c}$ states, $Z_{c}(4025)$ in $D^{*} D^{*}$ [5] and $Z_{c}$ (3885) in $D D^{*}$ mode [6]. Also $X(3872)$ is observed in $e^{+} e^{-} \rightarrow \gamma X(3872), X(3872) \rightarrow \pi^{+} \pi^{-} J / \psi[7]$. All the BESIII released paper in 2013 are shown in Fig. 1.

During 2013 and 2014, 1.8fb $\mathrm{fb}^{-1}$ scan XYZ data is taken at BESIII. With this scan data and previous data samples, BESIII published four paper about cross section measurements at this energy region and mainly for the Y sates study. A neutral counterpart of $Z_{c}(4020)$ observed in $\pi^{0} \pi^{0} h_{c}$ final states [8], 
discovery of a peak in the cross section line-shape of $e^{+} e^{-} \rightarrow \omega \chi_{c 0}$ [9], evidence of $e^{+} e^{-} \rightarrow \gamma \chi_{c 1}$ and $\gamma \chi_{c 2}$ [10], no significant signal of $Y(4140)$ is observed in $e^{+} e^{-} \rightarrow \gamma \phi J / \psi$ at $4.23 \tilde{4} .36 \mathrm{GeV}$ [11]. All the BESIII released paper in 2014 are shown in Fig. 2.
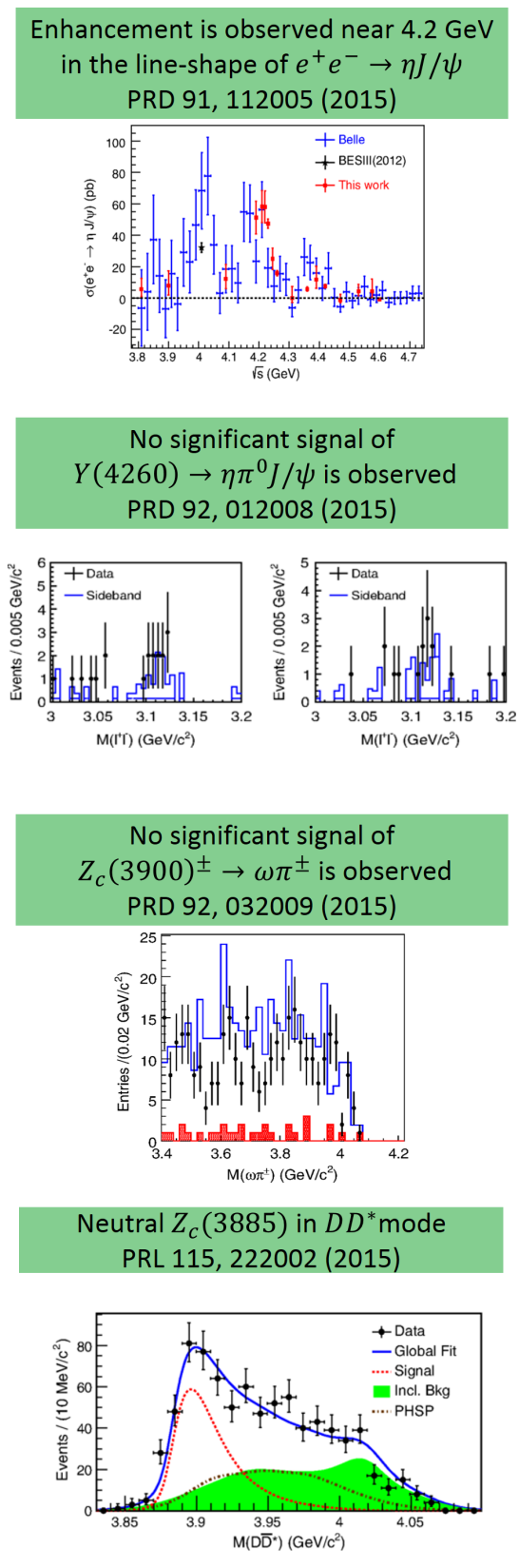

Figure 3. BESIII exotic hadron paper released in 2015.
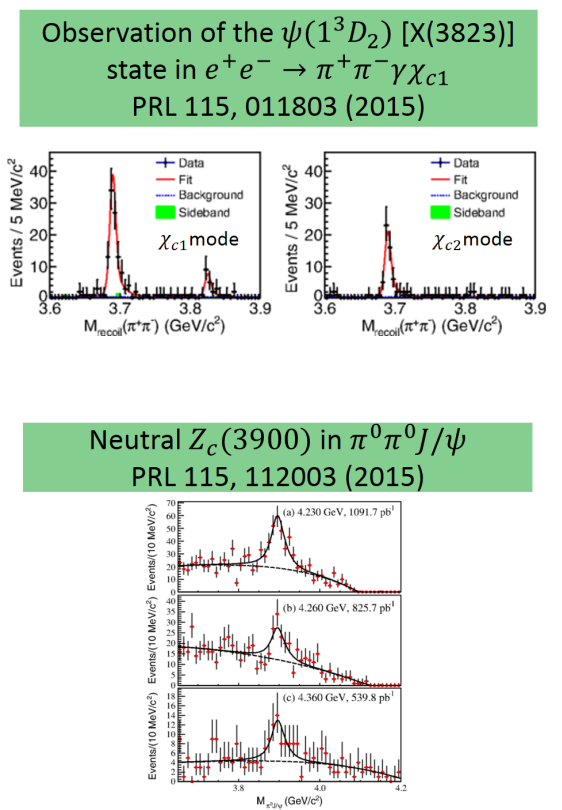

\section{Neutral $Z_{c}(4025)$ in $D^{*} D^{*}$ mode} PRL 115, 182002 (2015)
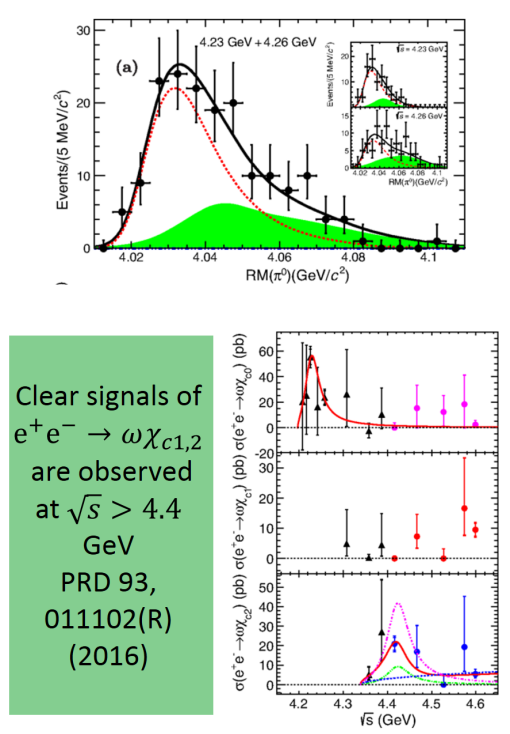
Mre more results came from BESIII in 2015. They are an enhancement is observed near $4.2 \mathrm{GeV}$ in the line-shape of $e^{+} e^{-} \rightarrow \eta J / \psi$ [12] observation of the $\psi\left(1^{3} D_{2}\right)[X(3823)]$ state in $e^{+} e^{-} \rightarrow \pi^{+} \pi^{-} \gamma \chi_{c 1}$ [13], in the cross section line-shape of $e^{+} e^{-} \rightarrow \eta \pi^{0} J / \psi$ and no significant signal of $Y(4260)$ is observed [14], neutral $Z_{c}(3900)$ is observed in $\pi^{0} \pi^{0} J / \psi$ final states [15], no significant signal of $Z_{c}(3900)^{ \pm} \rightarrow \omega \pi^{ \pm}$is observed [16], neutral $Z_{c}(4025)$ is observed in $D^{*} D^{*}$ mode [17], confirmation of a charged charmonium-like state $Z_{c}(3885)^{\mp}$ in $e^{+} e^{-} \rightarrow \pi^{ \pm}\left(D D^{*}\right)^{\mp}$ with double D tag [18], neutral $Z_{c}$ (3885) in $D D^{*}$ mode [19], clear signals of $e^{+} e^{-} \rightarrow \omega \chi_{c 1,2}$ are observed at $\sqrt{s}>4.4 \mathrm{Gev}$ [20]. All the BESIII released paper in 2015 are shown in Fig. 3.
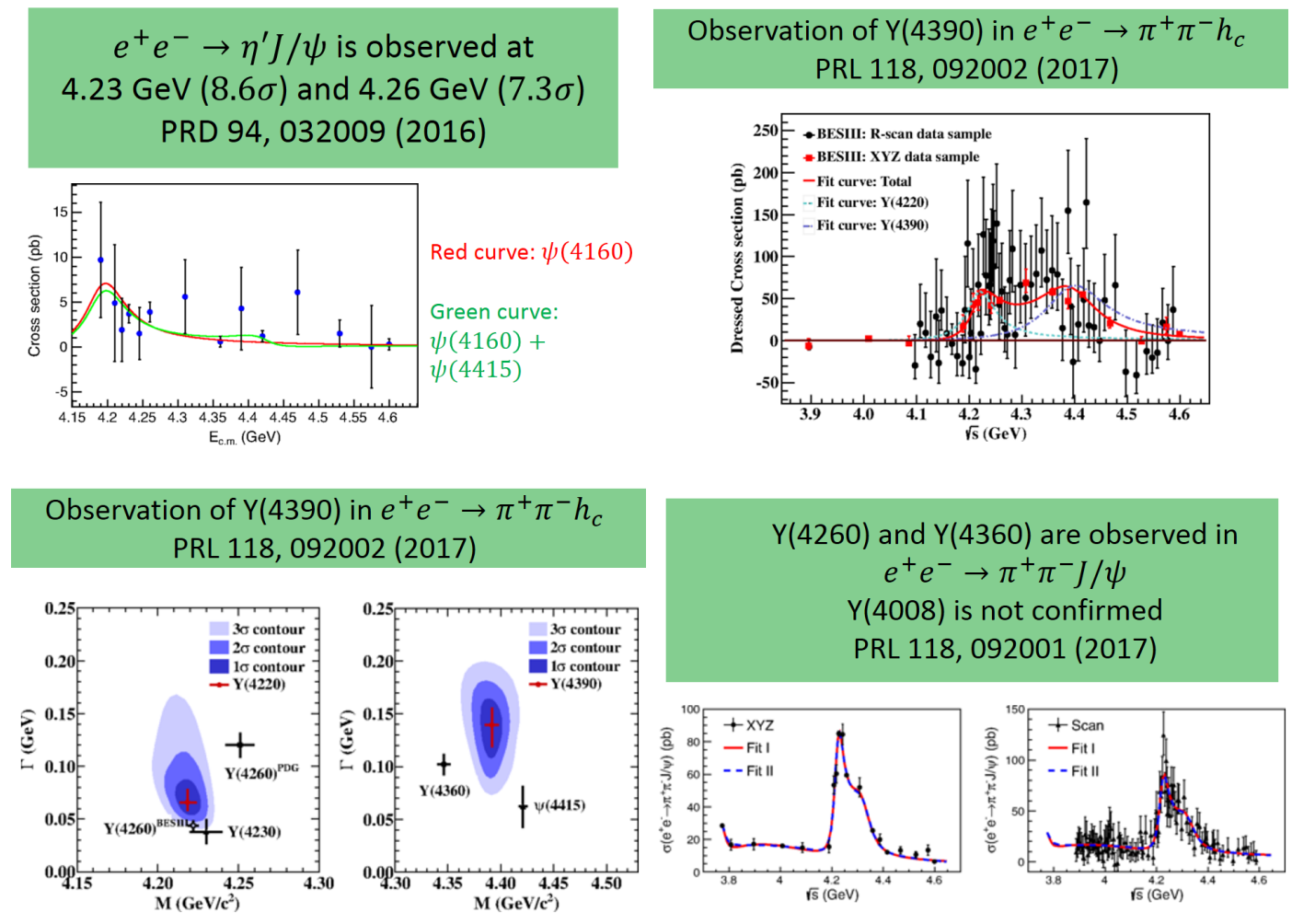

\section{$\mathrm{Y}(4260)$ and $\mathrm{Y}(4360)$ are observed in $e^{+} e^{-} \rightarrow \pi^{+} \pi^{-} J / \psi$ $\mathrm{Y}(4008)$ is not confirmed PRL 118, 092001 (2017)}

Figure 4. BESIII exotic hadron paper released in 2016.

In 2016, after released a cross section measurement of $e^{+} e^{-} \rightarrow \eta^{\prime} J / \psi$ [21], BESIII has accumulated $3.2 \mathrm{fb}^{-1}$ data at $4.18 \mathrm{GeV}$. Then it published two studies of Y states with hidden charm final states via cross section measurement. One process is $e^{+} e^{-} \rightarrow \pi^{+} \pi^{-} h_{c}$ [22] and the other is $e^{+} e^{-} \rightarrow \pi^{+} \pi^{-} J / \psi$ [23]. In both of them, two Y states are observed, in which one is a confirmation of previous discovery and the other one is new. And in the second analysis, $Y(4008)$ is not confirmed. All the BESIII released paper in 2016 are shown in Fig. 4. 

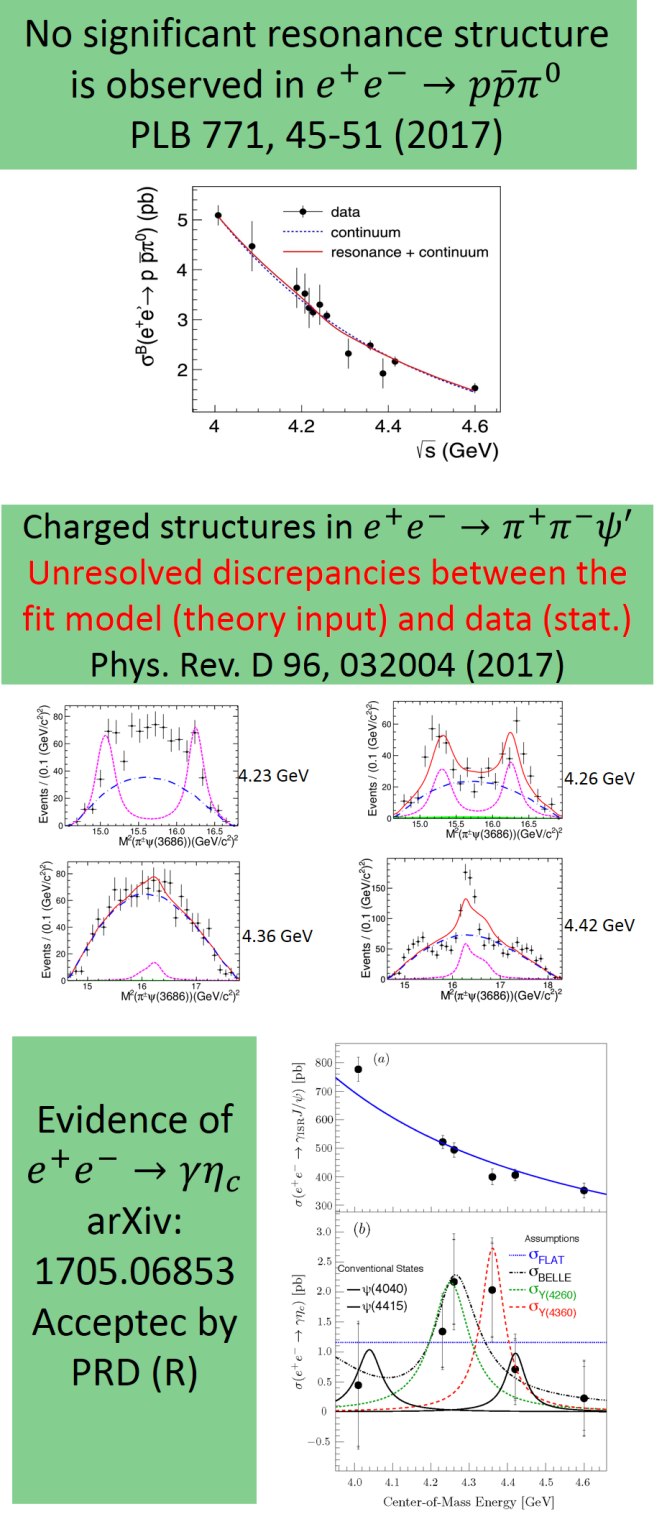

Cross section of $e^{+} e^{-} \rightarrow \pi^{+} \pi^{-} \psi^{\prime}$
Phys.
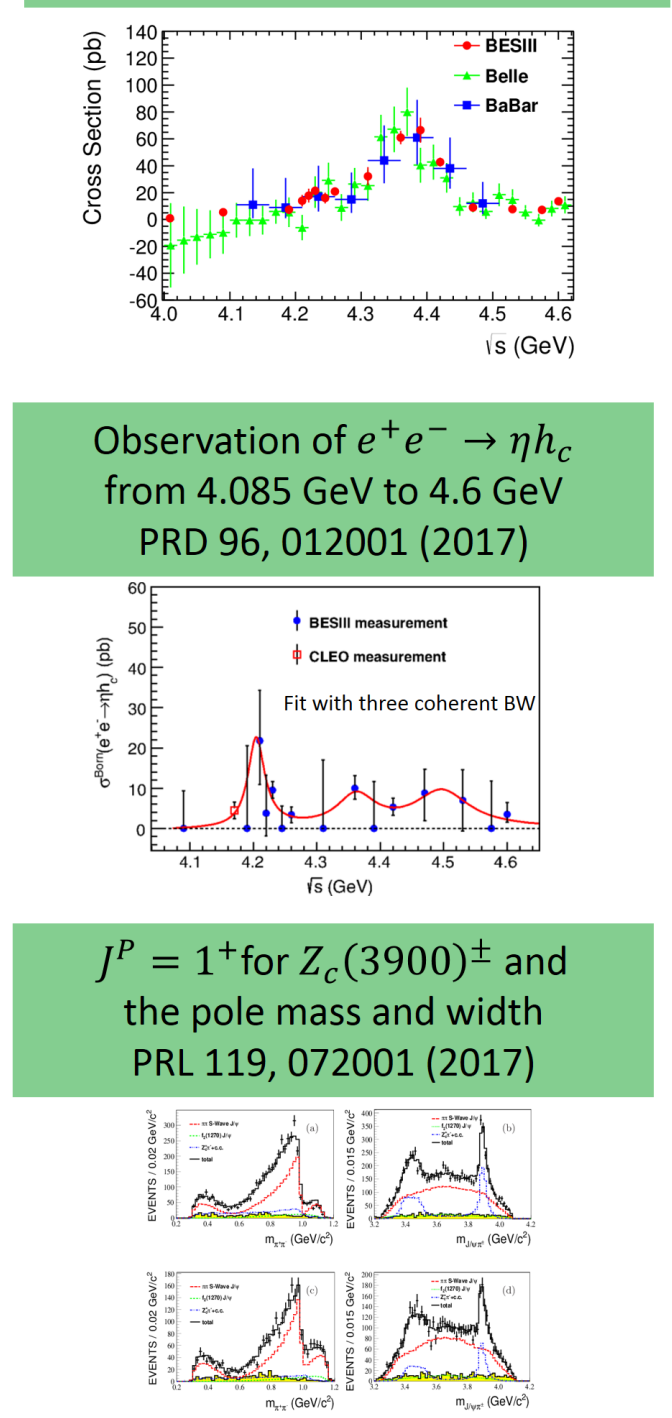

Figure 5. BESIII exotic hadron paper published in 2017.

In 2017, BESIII continues working on exotic hadron spectrum. It published the cross section measurement of $e^{+} e^{-} \rightarrow p \bar{p} \pi^{0}$ [24], in which no significant resonance structure is observed. And it has measured cross section and studied internal charged structures with $e^{+} e^{-} \rightarrow \pi^{+} \pi^{-} \psi(3686)$ [25], where complex structure is found and there is unresolved discrepancies between the fit model and data. It also has observed process $e^{+} e^{-} \rightarrow \eta h_{c}$ from $4.085 \mathrm{GeV}$ to $4.6 \mathrm{GeV}$ [26], evidence of $e^{+} e^{-} \rightarrow \gamma \eta_{c}$ [27], $J^{P}=1^{+}$for $Z_{c}(3900)^{ \pm}$and its pole mass and width [28]. And in this year, BESIII has collected more 
data for exotic hadron study with the scan energy from 4.19 to $4.28 \mathrm{GeV}$ and the step is $0.01 \mathrm{GeV}$. After that, there are some recent-released preliminary results: cross sections and neutral structure in $e^{+} e^{-} \rightarrow \pi^{0} \pi^{0} \psi(3686)$, structures in the line-shape of $e^{+} e^{-} \rightarrow K K J / \psi$, observation of $Y(4220)$ and $Y(4390)$ in $e^{+} e^{-} \rightarrow \pi^{+} D^{0} D^{*-}$ cross section between 4.05 and $4.60 \mathrm{GeV}$. All the BESIII published paper and released preliminary results in 2017 are shown in Figs. 5 and 6, respectively.

\section{Cross sections and neutral structure in $e^{+} e^{-} \rightarrow \pi^{0} \pi^{0} \psi(3686)$ \\ arXiv:1710.10740, submitted to PRL}
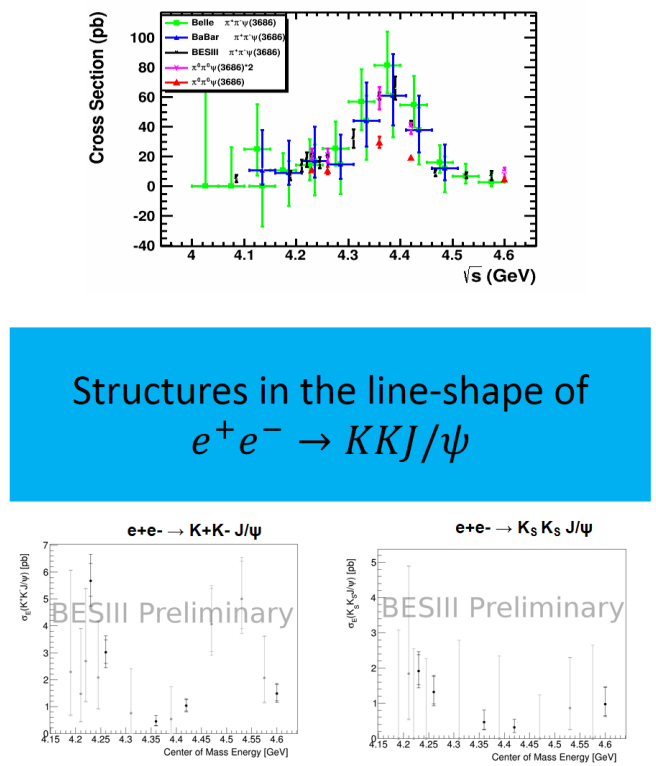

\section{Cross sections and neutral structure in $e^{+} e^{-} \rightarrow \pi^{0} \pi^{0} \psi(3686)$ arXiv:1710.10740, submitted to PRL}
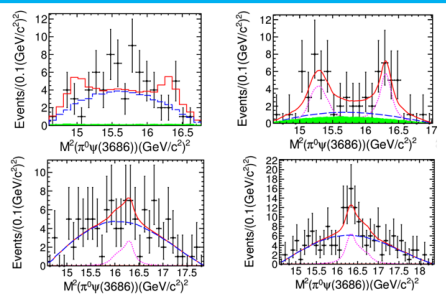

Observation of $\mathrm{Y}(4220)$ and $\mathrm{Y}(4390)$ in $e^{+} e^{-} \rightarrow \pi^{+} D^{0} D^{*-}$ cross section between 4.05 and $4.60 \mathrm{GeV}$

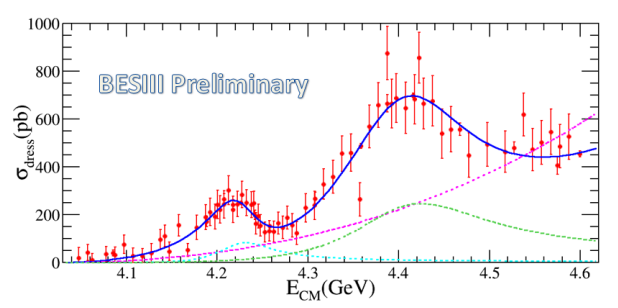

Figure 6. BESIII exotic hadron preliminary results released in 2017.

A simplified vision of these exotic hadron spectroscopy results is shown in Fig. 7, where all the $\mathrm{Y}$ or $\mathrm{Z}$ states with similar mass and with are combined into a single one. From this plot we may conclude the two $Z_{c}$ states, $Z_{c}(3900)$ and $Z_{c}(4020)$, have been established concretely by different isospin partners and different decays modes. The iso-spin symmetry is satisfied for the cross sections of charged and neutral structures. Meanwhile, two $Y$ states, $Y(4260)$ and $Y(4360)$, have been well established too by many different decay modes. 


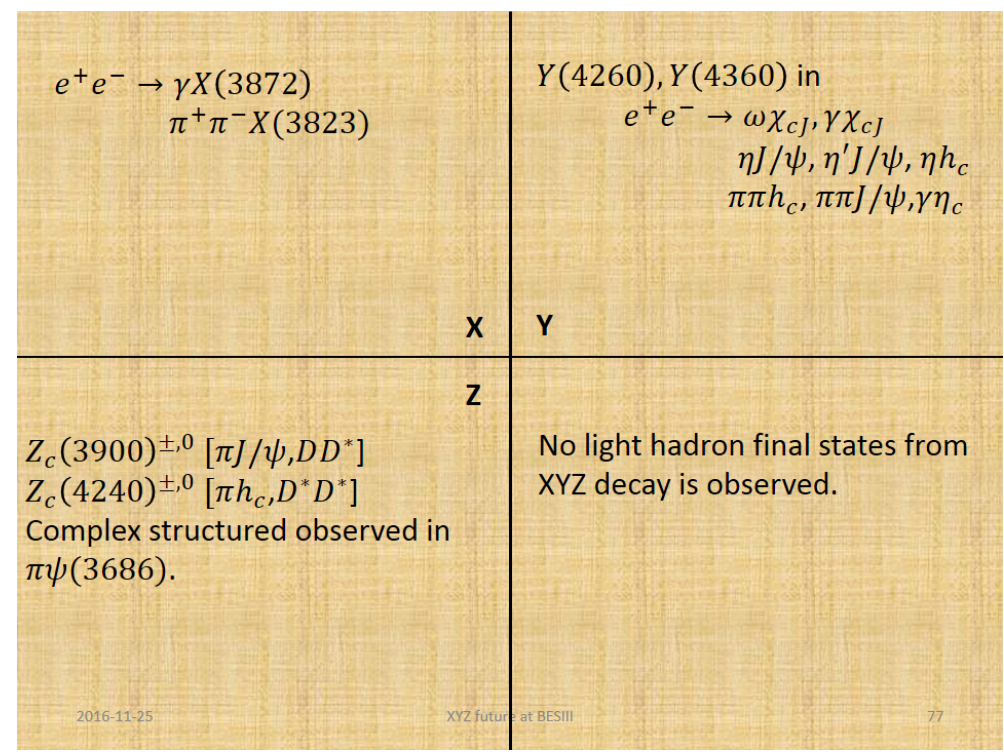

Figure 7. A very simplified vision of exotic hadron results at BESIII.

\section{Characteristics and trends in $\mathrm{XYZ}$ study at BESIII}

- Systematic

Ex.

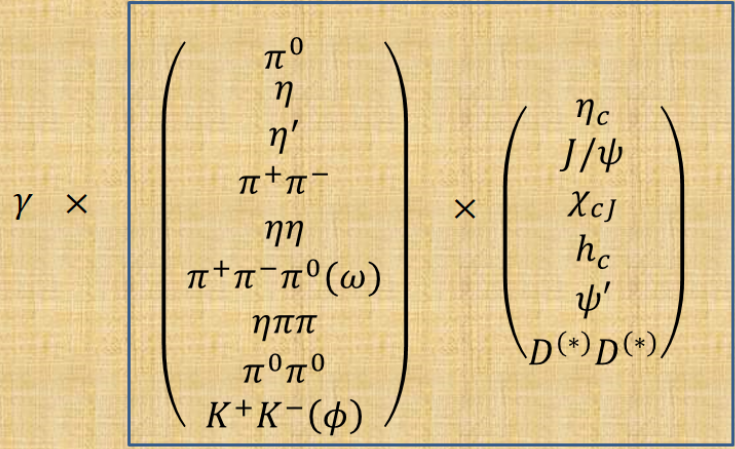

XYZ states at BESIII

Figure 8. One trend in BESIII measurement is a systematical exploration on all combinations of photon(s), light hadron(s), and hidden/open charm meson(s).

It seems fine for a somewhat good understanding of these exotic hadron states due to BESIII discoveries and measurements, but the problem is that this vision may be too simplified! Actually, many remained question need to be answered. For example, is there one or two states when two 
resonances takin similar mass and width? Complex internal structures are observed in $\pi \pi J / \psi$ and $\pi \pi \psi(3686)$ final states, how to describe them? Why some expected states and decay modes have not been observed? And how to explain some unexpected resonances and decay modes? Are there more exotic hadrons? What the relation between them and the previous observed ones? What is the nature of these exotic hadron states? Try to solve these problems and explore new features of the exotic hadrons, BESIII continue to work on the measurements of them and some features appear. One is the investigations are more systematical. As shown in Fig. 8, the topics are not selected as a random pick-up, but try to cover all the combinations of photon(s), light hadron(s) and hidden/open charm meson(s). It will provide us a broader and complete vision of this scenery. The other trend is that more complex and novel analysis methods are adopted in BESIII analyses, such as partial wave analysis (amplitude analysis), to extract more valuable information from data instead of a simple fit of the mass spectrum.

\section{Data taking plan for the $\mathrm{XYZ}$ particles at BESIII}

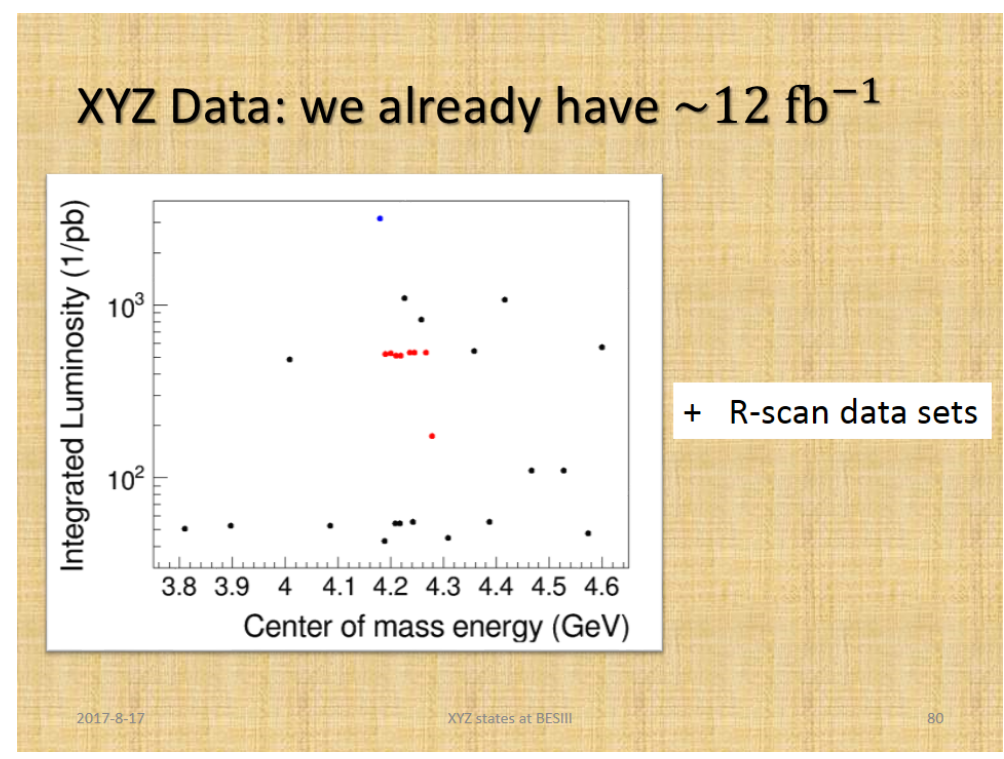

Figure 9. Integrated luminosity of data sets taken designedly for exotic hadron study at BESIII. The red points are scan data taken from $4.19 \mathrm{GeV}$ to $4.28 \mathrm{GeV}$ during early 2017 .

To understand these exotic hadrons, as shown in Fig. 9, BESIII has designedly collected more than $12 \mathrm{fb}^{-1}$ data, and plan to take more. From Fig. 9, we can see there are eight red points, that are corresponding to the scan data from 4.19 to $4.28 \mathrm{GeV}$ recently taken at BESIII during the early 2017. With them, more exotic hadron measurements, especially the cross section measurement with hidden or open charm meson are expected. The BESIII collaboration will investigate the line-shape of the cross section of processes with Y states, then help to distinguish nearby-resonances, precisely determine their mass and with, and shed light on their nature. Of course, more results on the $X$ and $\mathrm{Z}$ states are expected with the contribution of the newly increased luminosity. There was a "big plan" proposed by BESIII charmonium working group: start from $4.0 \mathrm{GeV}$ up to the maximum energy 
BEPCII can reach $(\leq 4.6) \mathrm{GeV}, 10 \mathrm{MeV}$ step (slight adjust near thresholds, skip points we have already collected large samples) scan is performed with $500 \mathrm{pb}^{-1} /$ point. Then we can expect $\sim 4.5 \mathrm{fb}^{-1}$ per year, a bit conservative than BEPCII design luminosity, and $4.6 \mathrm{GeV}$ can be reached in 2021 if we start at 2015. Now part of this "big plan" has been finished with scan data taken from 4.18 to $4.28 \mathrm{GeV}$ as well as other data taken at $4.36,4.42$, and $4.6 \mathrm{GeV}$, etc. Of course there are uncertainties and different options for the future plan. One possible choice is to take a few high statistics points (around 4.2, 4.3, and 4.4 GeV?), that primarily focus on further studies on $Z_{c}$ states. Or more high energy data (between 4.5 and $4.6 \mathrm{GeV}$ ?), that primarily focus on the line-shapes, $\Lambda_{c} \Lambda_{c}$ threshold, and more phase space. Or we can take data beyond $4.6 \mathrm{GeV}$, the present upper limit of BEPCII, to study higher mass exotic hadrons. Now the research and design of enhance the BEPCII upper limit energy is on-going. Or other plans may be based on suggestions from theorists, and specific predictions to the experimental results are extremely welcome.

\section{Summary}

BESIII has observed/measured many exotic hadron states. From the investigation of these hadron spectroscopy, some $Z_{c}$ and $Y$ states, $Z_{c}(3900 / 4020)$ and $Y(4260 / 4360)$ are solidly established based on the BESIII measurements. Our knowledge of these exotic states has been improved significantly. However, there are still many remaining puzzles. To solve them, BESIII plan to take more data in this energy region, and adopt/develop novel analysis tools to explore the hidden elements, and many exciting results are coming soon. With these efforts and inputs from our theorist friends, these exotic hadron states must be better understood.

\section{References}

[1] D. M. Asner et al., Int. J. Mod. Phys. A 24, S1 (2009) [arXiv:0809.1869 [hep-ex]].

[2] M. Ablikim et al. [BESIII Collaboration], Nucl. Instrum. Meth. A 614, 345 (2010) [arXiv:0911.4960 [physics.ins-det]].

[3] M. Ablikim et al. [BESIII Collaboration], Phys. Rev. Lett. 110, 252001 (2013) [arXiv:1303.5949 [hep-ex]].

[4] M. Ablikim et al. [BESIII Collaboration], Phys. Rev. Lett. 111, no. 24, 242001 (2013) [arXiv:1309.1896 [hep-ex]].

[5] M. Ablikim et al. [BESIII Collaboration], Phys. Rev. Lett. 112, no. 13, 132001 (2014) [arXiv:1308.2760 [hep-ex]].

[6] M. Ablikim et al. [BESIII Collaboration], Phys. Rev. Lett. 112, no. 2, 022001 (2014) [arXiv:1310.1163 [hep-ex]].

[7] M. Ablikim et al. [BESIII Collaboration], Phys. Rev. Lett. 112, no. 9, 092001 (2014) [arXiv:1310.4101 [hep-ex]].

[8] M. Ablikim et al. [BESIII Collaboration], Phys. Rev. Lett. 113, no. 21, 212002 (2014) [arXiv:1409.6577 [hep-ex]].

[9] M. Ablikim et al. [BESIII Collaboration], Phys. Rev. Lett. 114, no. 9, 092003 (2015) [arXiv:1410.6538 [hep-ex]].

[10] M. Ablikim et al. [BESIII Collaboration], Chin. Phys. C 39, no. 4, 041001 (2015) [arXiv:1411.6336 [hep-ex]].

[11] M. Ablikim et al. [BESIII Collaboration], Phys. Rev. D 91, no. 3, 032002 (2015) [arXiv:1412.1867 [hep-ex]]. 
[12] M. Ablikim et al. [BESIII Collaboration], Phys. Rev. D 91, no. 11, 112005 (2015) [arXiv:1503.06644 [hep-ex]].

[13] M. Ablikim et al. [BESIII Collaboration], Phys. Rev. Lett. 115, no. 1, 011803 (2015) [arXiv:1503.08203 [hep-ex]].

[14] M. Ablikim et al. [BESIII Collaboration], Phys. Rev. D 92, no. 1, 012008 (2015) [arXiv:1505.00539 [hep-ex]].

[15] M. Ablikim et al. [BESIII Collaboration], Phys. Rev. Lett. 115, no. 11, 112003 (2015) [arXiv:1506.06018 [hep-ex]].

[16] M. Ablikim et al. [BESIII Collaboration], Phys. Rev. D 92, no. 3, 032009 (2015) [arXiv:1507.02068 [hep-ex]].

[17] M. Ablikim et al. [BESIII Collaboration], Phys. Rev. Lett. 115, no. 18, 182002 (2015) [arXiv:1507.02404 [hep-ex]].

[18] M. Ablikim et al. [BESIII Collaboration], Phys. Rev. D 92, no. 9, 092006 (2015) [arXiv:1509.01398 [hep-ex]].

[19] M. Ablikim et al. [BESIII Collaboration], Phys. Rev. Lett. 115, no. 22, 222002 (2015) [arXiv:1509.05620 [hep-ex]].

[20] M. Ablikim et al. [BESIII Collaboration], Phys. Rev. D 93, no. 1, 011102 (2016) [arXiv:1511.08564 [hep-ex]].

[21] M. Ablikim et al. [BESIII Collaboration], Phys. Rev. D 94, no. 3, 032009 (2016) [arXiv:1605.03256 [hep-ex]].

[22] M. Ablikim et al. [BESIII Collaboration], Phys. Rev. Lett. 118, no. 9, 092002 (2017) [arXiv:1610.07044 [hep-ex]].

[23] M. Ablikim et al. [BESIII Collaboration], Phys. Rev. Lett. 118, no. 9, 092001 (2017) [arXiv:1611.01317 [hep-ex]].

[24] M. Ablikim et al. [BESIII Collaboration], Phys. Lett. B 771, 45 (2017) [arXiv:1701.04198 [hepex]].

[25] M. Ablikim et al. [BESIII Collaboration], Phys. Rev. D 96, no. 3, 032004 (2017) [arXiv:1703.08787 [hep-ex]].

[26] M. Ablikim et al. [BESIII Collaboration], Phys. Rev. D 96, no. 1, 012001 (2017) [arXiv:1704.08033 [hep-ex]].

[27] M. Ablikim et al. [BESIII Collaboration], Phys. Rev. D 96, no. 5, 051101 (2017) [arXiv:1705.06853 [hep-ex]].

[28] M. Ablikim et al. [BESIII Collaboration], Phys. Rev. Lett. 119, no. 7, 072001 (2017) [arXiv:1706.04100 [hep-ex]]. 